\title{
Atomic-Scale Observation of Lithiation Reaction Front in Single
}

\section{$\mathrm{SnO}_{2}$ Nanowire}

Anmin $\mathrm{Nie}^{1}$, Liyong $\mathrm{Gan}^{2}$, Yingchun Cheng ${ }^{2}$, Hasti Asayesh Ardakani ${ }^{1}$, Qianqian $\mathrm{Li}^{3}$, Cezhou Dong ${ }^{3}$, Runze $\mathrm{Tao}^{4}$, Farzad Mashayek ${ }^{5}$, Hongtao Wang $^{3}$, Udo Schwingenschlogl ${ }^{2}$, Robert $\mathrm{Klie}^{4}$, Reza Shahbazian-Yassar ${ }^{{ }^{*}}$

${ }^{1}$ Department of Mechanical Engineering-Engineering Mechanics, Michigan

Technological University, 1400 Townsend Dive, Houghton, Michigan 49931, United States

${ }^{2}$ Department of Physical Science and Engineering, King Abdullah University of Science \& Technology, Thuwal, 23955-6900, Kingdom of Saudi Arabia ${ }^{3}$ Institute of Applied Mechanics, Zhejiang University, Hangzhou, P. R. China 310027

${ }^{4}$ Department of Physics, University of Illinois at Chicago, Chicago, Illinois 60607, United States

${ }^{5}$ Mechanical and Industrial Engineering Department, University of Illinois at Chicago, Chicago, Illinois 60607, United States

Development of new high-performance electrode materials for in lithium ion batteries (LIBs), especially the anode materials, has been under intense research during the past decade. The design of new electrode materials to a great extent depends on how the lithiation front propagates into the anode material. Therefore, revealing the atomic scale lithiation mechanism is central to unfolding the performance of electrode materials during the operation of LIBs. Although recent studies of in situ TEM LIBs ${ }^{[1-2]}$ have revealed morphology evolution, structure and chemical changing in the anode materials during the electrochemical reaction, there is currently lack of critical knowledge about the atomistic mechanisms of dynamical lithiation.

In the present work, taking advantage of an aberration-corrected scanning transmission electron microscopy (STEM), we show that the dynamic lithiation process of anode materials with atomic resolution can be revealed. Atomically resolved imaging of the lithiation process in $^{\mathrm{SnO}_{2}}$ nanowires illustrated that at the very initial stage of lithiation, lithium ions preferred to diffuse along [001] direction in the $\{200\}$ planes, which introduced the lattice expansion and dislocations. The

movement, reaction and generation of $\boldsymbol{b}=[111]$ mixed dislocations effectively facilitated lithium ion insertion into the crystalline interior. At the later stages of lithiation, the Li-induced amorphization of rutile $\mathrm{SnO}_{2}$ and the formation of crystalline Sn particles in an amorphous matrix were observed. In situ high resolution TEM imaging revealed that the morphological evolution and mobility of the interface between the crystalline $\mathrm{Sn}$ and amorphous $\mathrm{Li}_{\mathrm{x}} \mathrm{Sn}$ were orientation-dependent during lithiation. 
Reference

[1] Huang, J. Y.; Zhong, L.; Wang, C. M.; Sullivan, J. P.; Xu, W.; Zhang, L. Q.; Mao, S. X.; Hudak, N. S.; Liu, X. H.; Subramanian, A. Science 2010, 330, 1515-1520. [2] Wang, C. M.; Xu, W.; Liu, J.; Choi, D.; Arey, B.; Saraf, L. V.; Zhang, J.; Yang, Z.; Thevuthasan, S.; Baer, D. R. J. Mater. Res. 2010, 25, 1541-1547.

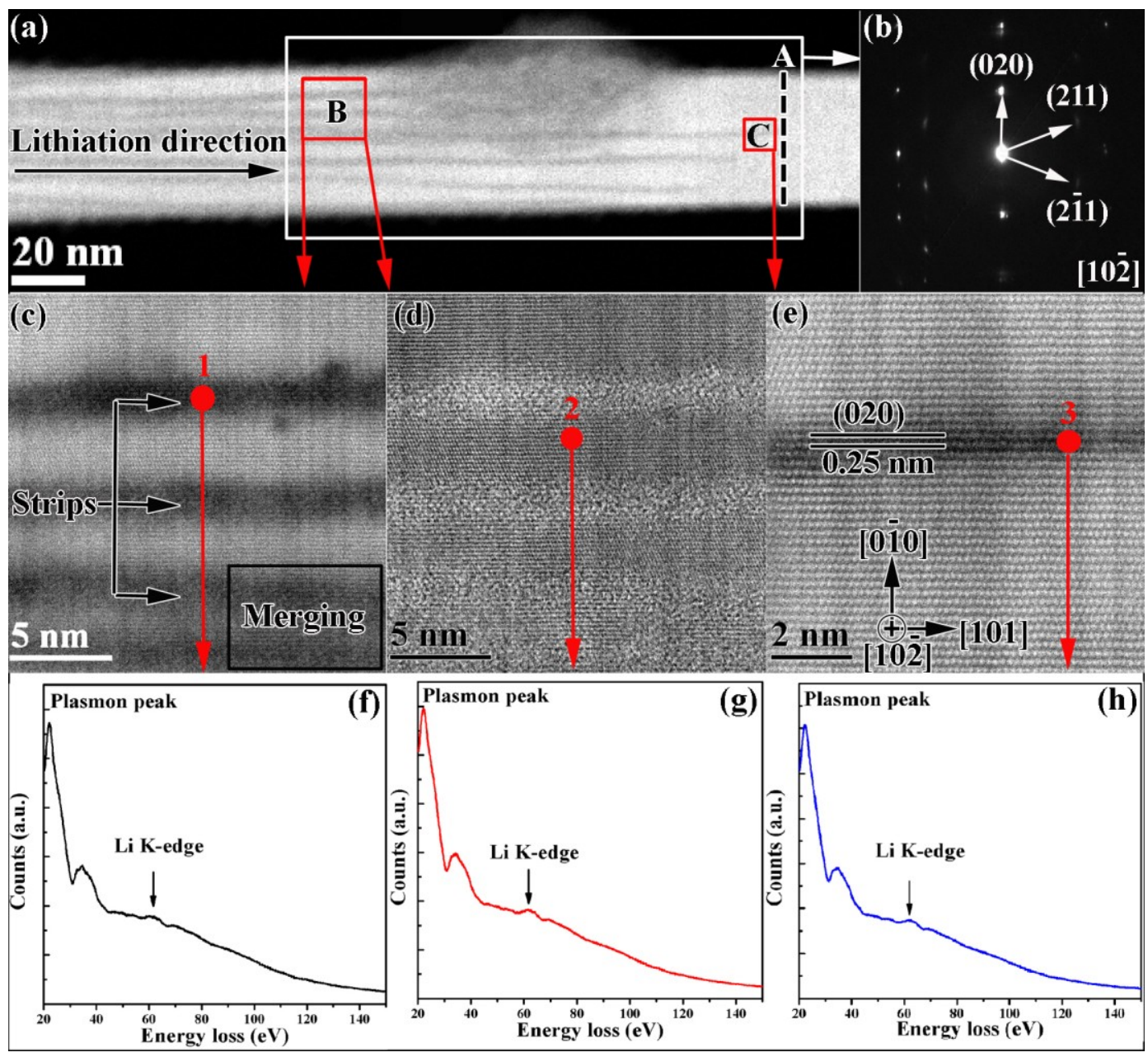

Figure 1 (a) HAADF image taken from the reaction front of a partially lithiated $\mathrm{SnO}_{2}$

nanowire. (b) The corresponding [10를 zone axis SAED pattern taken from the area marked as A. (c) Higher magnification HAADF of the area marked as B in (a), and (d) is the corresponding bright field image showing lithiation strips along the $\mathrm{SnO}_{2}$ nanowire. (e) An atomic resolution HAADF image taken from the area near the tip of one lithiation strip. (f)-(h) EELS spectra taken from the different areas (marked by red spots as 1, 2, 3 in STEM images (c-e)) showing Li-K edge in the $\mathrm{SnO}_{2}$ nanowire, respectively. 